\title{
Peptic Ulcer in Veterinary Medicine: A Mini Review
}

\author{
Ebbo AA ${ }^{1 *}$, Bello A², Ismail MS1 and Magami IM³ \\ ${ }^{1}$ Department of Veterinary Pharmacology and Toxicology, Usmanu Danfodiyo \\ University, Sokoto, Nigeria \\ ${ }^{2}$ Department of Veterinary Anatomy, Usmanu Danfodiyo University, Sokoto, Nigeria \\ ${ }^{3}$ Department of Biological Sciences (Zoology Unit), Usmanu Danfodiyo University, \\ Sokoto, Nigeria
}

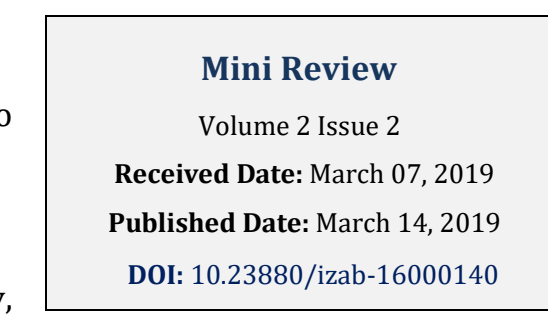

*Corresponding author: Ebbo AA, Department of Veterinary Pharmacology and Toxicology, Usmanu Danfodiyo University, Sokoto, Nigeria, Email: aaliyuebbo@yahoo.co.uk

\section{Abstract}

Ulceration and discontinuity of the gastrointestinal mucosal can be a consequence of several drugs and diseases in small animals. As a result, gastroprotectant therapies are widely used in veterinary patients. Gastrointestinal ulcers can occur independently or as a complication of many systemic diseases hence have a significance importance to clinician in understand the etiological factors, pathophysiology for their effective treatment and early prophylaxis.

Keywords: Gastrointestinal tract, duodenum, ulcer, Veterinary Medicine

\section{Introduction}

Peptic ulcers can be sores that develop in the lining of the lower esophagus, stomach or small intestine. They're usually formed as a result of excessive gastric acid and inflammation caused by the pathogenic bacteria H. pylori, as well as from erosion from excessive stomach and intestinal motility. Peptic ulcers are common health problem associated with human and animals.

\section{Description of Peptic Ulcer Disease}

Peptic Ulcer Disease (PUD) also known as peptic ulcer or stomach ulcer is a break in the lining of the stomach, first part of the small intestine, or occasionally the lower esophagus [1]. It occurs due to the loss of the balance between aggressive and defensive factors of the gastric and duodenal mucosae. Aggressive factors against gastric mucosa which include acid, pepsin, Helicobacter pylori, non-steroidal anti-inflammatory drugs (NSAIDs) and ethanol; while local mucosal defensive factors include bicarbonate, mucus secretion, blood flow, cellular regeneration, and endogenous protective agents like prostaglandins and epidermal growth factor [2]. It is characterized by lesions in the lining of the upper gastro intestinal tract, which often compromise all layers of the mucosa, piercing the tissue and provoking bleeding. Peptic ulcer disease most often presents as either gastric or duodenal ulcer [3]. Racial difference has also been observed, and in Africa duodenal ulcers are found to be rare in black people, but in the United States the incidence is the same for blacks and whites; regarding gender, there is predominance of ulcers in males [4].

Histologically, peptic ulcer is identified as necrosis of the mucosa which produces lesions equal to or greater than $0.5 \mathrm{~cm} \mathrm{(1/5");} \mathrm{it} \mathrm{is} \mathrm{the} \mathrm{most} \mathrm{common} \mathrm{ulcer} \mathrm{of} \mathrm{an} \mathrm{area}$ of the gastrointestinal tract that is usually acidic and thus extremely painful [5]. Clinical manifestations are characterized by epigastric discomfort, burning or severe and continuous pain, which tends to be worse at night. Pain usually happens one to three hours after eating, and may be followed by nauseas, vomiting, gastrointestinal discomfort, flatulence, and significant loss of body weight. 
The histological analysis of the gastric mucosa showed lymphoid follicles in $28 \%$ of the patients, being associated with nodular lesions in the gastric antrum in $31 \%$ of the cases [6].

Gastric erosions are divided into three categories: complete erosion, incomplete erosion and hemorrhagicerosive gastritis with the last of these three consisting of numerous, pinpoint-sized hemorrhages' on the mucosa and in addition within the mucosa and sub mucosa, erythrodiapedesis and engorged blood vessels [7] Unrecognized hematologic manifestations with H. pylori infection include: autoimmune neutropenia, antiphospholipid syndrome, plasma cell dyspraxia, monoclonal gammopathy of undetermined significance, multiple myeloma and other possible associations with diseases such as leukemia and hemorrhagic manifestations with a hematological origin, congenital coagulopathies and bleeding disorders [8].

\section{Causes of Peptic Ulcer Disease}

The etiology of PUD includes; Helicobacter pylori infection, NSAIDs, smoking, alcohol, bile-acids, steroids, stress, and changes in gastric mucin consistency. Other causes include Behcet's disease, Zollinger-Ellison syndrome, Cohn disease, liver cirrhosis, coronary heart disease, and inflammation of the stomach lining or gallbladder [1]. Helicobacter pylori infection and the use of non steroidal anti-inflammatory drugs (NSAID) are considered the main risk to develop peptic ulcer disease where PUD can occur in the absence of Helicobacter pylori infection and or use of NSAIDs [3]. Recently, evidence has been found that Epson- Barr virus (EBV) reactivation increases the risk of developing pre malignant and malignant gastric lesions [3]. Peptic ulcer disease may be due to any of the following: Helicobacter pylori infection, drugs, lifestyle factors, severe physiologic stress, and hyper secretion of gastric acids, genetic factors and so on [9].

\section{NSAID Induced Ulcers}

The pathogenesis of NSAIDs-induced gastric ulceration involves the block of cyclo-oxygenase (COX) activity that leads to lower mucus and bicarbonate secretions, decreased mucosal blood flow, neutrophil infiltration, alteration of micro vascular structures, increase in acid and pepsinogen secretion [10]. In addition, increased production of reactive oxygen species (ROS), increased lipid peroxidation and neutrophil infiltration have been demonstrated to play a role in the pathogenesis of NSAIDs-induced ulcers, including the aspirin-induced ulcer [2]. NSAIDs exert anti-inflammatory, antipyretic and analgesic effects by inhibiting cyclooxygenase (COX-1 and COX-2) enzymes which are responsible for the production of prostaglandins, prostacyclin's and thromboxane's. Risk factors associated with gastro-duodenal bleeding in patients on NSAID therapy include: age, prior peptic ulcer and co-medication with anti-platelet agents (eg. anticoagulants), glucocorticoids and selective serotonin reuptake inhibitors [11].

Conventional NSAIDs cause non-selective inhibition of cyclooxygenase which leads to reduction of bicarbonate secretion and reduced mucus production. Coupled with it is vasoconstriction that occur causing hypoxia and consequent formation of ulcer. Most NSAIDs are weak organic acids with a low $\mathrm{pKa}$, remaining unionized in the stomach and are absorbed appreciably in the stomach. Once they breach the cell membranes of stomach cells and reach within, they encounter a basic $\mathrm{pH}$ [12]. Peptic ulcers are defects in the gastric or duodenal mucosa that extends through the muscular is mucosa. The epithelial cells of the stomach and duodenum secrete mucus in response to irritation of the epithelial lining as a result of cholinergic stimulation. The superficial portion of the gastric and duodenal mucosa exists in the form of a gel layer, which is impermeable to acid and pepsin [13]. Other gastric and duodenal cells secrete bicarbonate, which aids in buffering the acid that lies beneath the mucosa. Prostaglandins of the E type (PGE) have an important protective role, because PGE increases the production of both bicarbonate and the mucous layer [9].

In the event of acid and pepsin entering the epithelial cells, additional mechanisms are in place to reduce injury; within the epithelial cells, ion pumps in the basolateral cell membrane help to regulate intracellular $\mathrm{pH}$ by removing excess hydrogen ions [14]. Through the process of restitution, healthy cells migrate to the site of injury. Mucosal blood flow removes acid that diffuses through the injured mucosa and provides bicarbonate to the surface epithelial cells [9].

\section{Smoking as a Cause of Ulcer}

Although some studies have found correlations between smoking and ulcer formation others have been more specific in exploring the risks involved and have found that smoking by itself may not be much of a risk factor unless associated with Helicobacter pylori infection [5]. Normally, acid starts breaking food down as it hits the stomach, priming the food for the more powerful digestive enzymes it will encounter further down the 


\section{International Journal of Zoology and Animal Biology}

digestive tract. Whatever acid isn't absorbed is neutralized by sodium bicarbonate, a natural antacid which occurs in the duodenum [15]. Smoking increases the stomach acid and reduces the bicarbonate level in the duodenum making it susceptible to ulcer [16]. Experimental findings suggest that cigarette smoking increases xanthine oxidase activity, leukotriene's, and nitric oxide production and also neutrophil infiltration in the gastric mucosa while on the other hand, it reduces blood flow, prostaglandin production, epithelial cell proliferation, and formation of blood vessels in tissues [17].

\section{Stress as a Cause of Ulcer}

'Stress-related mucosal damage' (SRMD) is a broad term used to describe the spectrum of pathology attributed to the acute, erosive, inflammatory insult to the upper gastrointestinal tract associated with critical illness [18]. Stress ulcers develop as a result of major physiological stress occurring in the fundus of the stomach and proximal duodenum and are caused by localized ischemia, tissue acidosis and the presence of bile salt in the stomach of patients with decreased gastrointestinal motility [19]. Ordinary peptic ulcers are found commonly in the gastric antrum and the duodenum whereas stress ulcers are found commonly in fundic mucosa and can be located anywhere within the stomach and proximal duodenum [20].

\section{Genetic Cause of Ulcer}

Although there are believed to be several genetic risk factors for peptic ulcer such as polymorphisms in immunoregularatory genes, only a few studies have addressed genetic factors in gastric erosion; where the G308A polymorphism of TNF-alpha, which causes elevated expression of the TNF- $\alpha$ protein, has been associated with erosive gastritis [7]. Much interest has been shown in the hereditary aspect of peptic ulcer but there is still doubt about its importance. This is not surprising because it was difficult to be certain of the diagnosis of an ulcer before radiology was developed and because the prevalence of the condition makes the chance association of several cases in a family probable [21].

\section{Helicobacter Pylori Infection as a Cause of Ulcer}

Helicobacter pylori is a spiral-shaped bacterium that is found in the gastric mucous layer or adherent to the epithelial lining of the stomach which causes more than $90 \%$ of duodenal ulcers and up to $80 \%$ of gastric ulcers [22]. Helicobacter pylori bacteria adhere to the gastric mucosa via the presence of an outer inflammatory protein and a functional cytotoxin-associated gene island in the bacterial chromosome increasing its virulence and probably ulcerogenic potential [13]. Helicobacter pylori usually settles into the body during childhood, but ulcers rarely develop before the age of 30 , and only one in five people with Helicobacter pylori infections ever develop an ulcer [20].

Helicobacter pylori may also have a role in uninvestigated and functional dyspepsia, ulcer risk in patients taking low-dose aspirin or starting therapy with a non-steroidal anti-inflammatory medication, unexplained iron deficiency anemia, and idiopathic thrombocytopenic purpura [23]. Infection with Helicobacter pylori is believed to occur mostly during childhood but the mode of transmission is not yet understood. It is believed that infection most likely occurs through contact with contaminated water, saliva, vomit or stool of infected persons [24].

\section{Conclusive Management of Ulcer}

Eradication of Helicobacter pylori is recommended in all patients with PUD [25]. The first-line therapy should have an eradication rate of more than $80 \%$ using a sevento ten-day triple drug regimen consisting of a proton pump inhibitor, amoxicillin $1 \mathrm{~g}$, and clarithromycin 500 mg (Biaxin) twice daily has long been the first-line therapy to eradicate Helicobacter pylori [26]. Most commonly used drugs for ulcer management include: antacids, H2-blockers, proton pump inhibitors which all act by reducing the aggressive factors with various reports showing that these commonly used drugs for peptic ulcers have danger of drug interaction, adverse effects and increased incidence of relapses during therapy $[27,28]$.

It is worthy to note that most of the gastrointestinal lesions heal as a result of self-regeneration or selfrenewal process within the body but some, however, develop serious lesions requiring clinical attention should be sought. Ulcer prophylaxis should be considered in the patients who are taking NSAIDs, but continue to smoke or use alcohol. Those patients who have ulcers but are not taking NSAIDs should receive antiulcer drugs like $\mathrm{H} 2$ blockers [12]. There are some drugs called cytoprotectives which enhance mucosal protection mechanism and provide a physical barrier over the surface of ulcer. Examples of such drugs are sulfa crate, carbenoxolone and bismuth chelate. Sulfa crate is a complex of aluminum hydroxide and sulphated sucrose which polymerizes at a $\mathrm{pH}$ of less than 4 . It acts by the 


\section{International Journal of Zoology and Animal Biology}

sulphated group binding to proteins in ulcerated tissues protecting ulcers from acid and pepsin. Carbenoxolone promotes production of mucus which lines and protects the gastric mucosa. Bismuth chelate acts by coating ulcer base, absorbing pepsin and enhancing local prostaglandin synthesis [10].

\section{References}

1. Murtaza M, Jayaram M, Muiandy RK, Fredie R, Sein MM, et al. (2015) Risk Factors, Diagnosis and Management of Peptic Ulcer Disease. IOSR Journal of Dental and Medical Sciences 4(7): 40-46.

2. Vivian MC, Yazmin RC, Yohami PG, Amber OY, Sonia JD, et al. (2011) Effects of Grape seed Extract, Vitamin $\mathrm{C}$ and Vitamin E on Ethanol and Aspirin- Induced Ulcers. Gastroenterology 35(1): 41-60.

3. Maria GMC, Javier T, Lourdes FL, Ricardo CT, Margarita CP, et al. (2015) Epson-Barr Virus Association with Peptic Ulcer Disease. Anal cell Pathol 2015: 164840 .

4. Vomero ND, Calpo E (2014) Nutritional Case in Peptic Ulcer. ABCD Arq Bras Cir Dig 27(4): 298-302.

5. Ifeanyi ED, Sunday GA (2014) Some Hematological and Biological Parameters in Peptic Ulcer Patients in Umudike, Abia State, Nigeria. World Journal of Pharmacy and Pharmaceutical Sciences 3(4): 294302.

6. Elizabete $\mathrm{K}$, Rodrigo SM, Jacqueline AF, Francy RSP (2004) Clinical and Histological Features of Duodenal Ulcers in Children and Adolescents. Journal de Pediatria 80(4): 321-325.

7. Toljamo K (2012) Gastric Erosions-Clinical Significance and Pathology. Acta University Ouluensis D 1152.

8. Maya GC (2014) Hematologic Manifestations of Helicobacter pylori Infection. World Journal of Gastroenterology 20(36): 12818-12838.

9. Anand BS, Philip OK (2018) Peptic Ulcer Disease. American Academy of Family Physicians 12(2): 102115.

10. Aliyu YO (2011) Nonsteroidal Anti-inflammatory drugs. In: Veterinary Pharmacology, $1^{\text {st }}$ (Edn.), pp: 123-124.
11. Marino V, Thomas W, Peter M (2010) Non-steroidal Anti-inflammatory Drug Induced Gastro duodenal Bleeding: Risk Factors and Prevention Strategies. Pharmaceuticals 3(7): 2225-2237.

12. Vikas D, Sindhu S, Swati P, Atul C, Kuljeet SA (2003) Non-steroidal Drug Toxicity: Mechanism and Management. Journal Indian Academy of Clinical Medicine 4(4): 315-322.

13. Ramakrishnan K, Robert CS (2007) Peptic Ulcer Disease. American Academy of Family Physicians 76(7): 1005-1012.

14. Subudhi BB, Sahoo SP, Sahu PK (2016) Drug Development Strategies against Peptic Ulcer. Journal of Gastroduodenal and Digestive System 6: 398.

15. Norra M (2009) Smoking and Ulcers. Everyday Digestive Health Management.

16. Kerama SK, Faith AO, David GN, Eric MG, Stanley NN, et al. (2014) Risk Factors and Management of Stress Ulcers in the Critical Care Unit in a Kenyan Referral Hospital. African Journal of Pharmacology and Therapeutics 3(3): 51-61.

17. Karvonen AL, Lehtola J (1983) Gastric Mucosal Erosions; a Clinical History and Findings and the Possible Role of Herpes simplex Infection in Aetiology. Ann Clin Res 15(4): 137-141.

18. Plummer PM, Annika RB, Adam MD (2014) Stress Ulceration: Prevalence, Pathology and Association with Adverse Outcomes. Annual Update in Intensive Care and Emergency Medicine 18: 213.

19. Samuel L (2014) Peptic Ulcer and Stress. The Encyclopedia of health and Behavior, Sage Publications 152: 829-833.

20. Susan L, Sigurd A, Janice KKG, Andre D (1999) Stress and Peptic Ulcer Disease. Journal of American Medical Association 281(1): 10-11.

21. Richard D, Buch J (1950) Hereditary Factors in Peptic Ulcer. Medical Research Council Memorandum, to be published.

22. Mitsuru M, Akira U, Toshikatsu O, Mitasuka, Yukata K (2011) Stress Induced Hemorrhagic Gastric Ulcer After Successful Helicobacter Pylori eradication: Two Case Reports. J Med Case Rep 5: 252. 


\section{International Journal of Zoology and Animal Biology}

23. William DC, Grigorios IL, Colin WH, Steven FM (2017) Treatment of Helicobacter pylori Infection. American Medical Journal Gastroenterol 112: 212-238.

24. Kurata JH, Haile BM (2014) Epidemiology of Peptic Ulcer Disease. American Journal of Epidemiology 37: 40-42.

25. Huang TC, Chia LL (2014) Diagnosis, Treatment and Outcome in Patients with Bleeding Peptic Ulcers and Helicobacter pylori Infections. Biomed Res Int 2014: 658108 .
26. Julia F, Alfred CG (2015) Diagnosis and Treatment of Peptic Ulcer Disease and H. pylori Infection. American Academy of Family Physicians 91(4): 236-242.

27. Tandel KR, Shah BK (2012) Evaluation of Gastric Antiulcerogenic Action of Vegetable Plantain Banana (Musa sapientum var paradasiaca) in Aspirin plus Pylorus Ligated Albino Rats. International Journal of pharmaceutical sciences and Research 3(11): 43874393.

28. Debjit B, Kumar SKP, Duraivel S, Umadevi M (2012) Traditional and Medicinal Uses of Banana. Journal of Pharmacognosy and Phytochemistry 1(3): 11-14. 